TITLE:

\title{
Molecular dynamics simulation of the break of magnesium nanowires
}

$\operatorname{AUTHOR}(\mathrm{S})$ :

Takahashi, Atsushi; Kurokawa, Shu; Sakai, Akira

\section{CITATION:}

Takahashi, Atsushi ... [et al]. Molecular dynamics simulation of the break of magnesium nanowires. physica status solidi (b) 2014, 251(7): 13631371

ISSUE DATE:

2014-05-13

URL:

http://hdl.handle.net/2433/200758

\section{RIGHT:}

This is the peer reviewed version of the following article:Takahashi, A., Kurokawa, S. and Sakai, A. (2014), Molecular dynamics simulation of the break of magnesium nanowires. Phys. Status Solidi B, 251: 1363-1371, which has been published in final form at http://dx.doi.org/10.1002/pssb.201350245. This article may be used for non-commercial purposes in accordance with Wiley Terms and Conditions for Self-Archiving.; This is not the published version. Please cite only the published version.; この論文は出版社版でありません。引用の際には出版社版をご確認ご利用ください。 


\title{
Molecular dynamics simulation of the break of magnesium nanowires
}

\author{
Atsushi Takahashi, Shu Kurokawa, Akira Sakai \\ Department of Materials Science and Engineering, Kyoto University, Sakyo-ku, Kyoto 606-8501, Japan \\ Received XXXX, revised XXXX, accepted XXXX \\ Published online XXXX
}

Key words: molecular dynamics simulation; contact break; magnesium nanowires

${ }^{*}$ Corresponding author: e-mail sakai.akira.4z@kyoto-u.ac.jp, Phone/FAX: +81-75-753-4833

We carried out molecular dynamics simulations of the tensile break of $\mathrm{Mg}$ nanowires at $4 \mathrm{~K}$ and $300 \mathrm{~K}$ and examined how the minimum cross section $S_{m}$ (measured by number of atoms) of a constrictive neck diminishes with nanowire stretching. For both [0001]- and [0110]-oriented nanowires, the histogram of $S_{m}$ at $4 \mathrm{~K}$ shows a peak at $S_{m}=1$. This result indicates that $\mathrm{Mg}$ nanowires can be reduced to single-atom contacts at $4 \mathrm{~K}$ in agreement with experimental observations. At the beginning of stretching, the tensile force of $\mathrm{Mg}$ nanowires at $4 \mathrm{~K}$ is much larger than that at $300 \mathrm{~K}$, but the forces at $4 \mathrm{~K}$ and $300 \mathrm{~K}$ become comparable in later deformation stages. We observed that the stretching of $\mathrm{Mg}$ nanowires at $300 \mathrm{~K}$ often produces long icosahedral nanowires similar to the one reported in the stretching simulations of some FCC nanowires. These icosahedral nanowires yield a large peak at $S_{m}=5$ in the $S_{m}$ histogram at $300 \mathrm{~K}$. It is found that this $S_{m}=5$ peak depends on the initial size of nanowires and decreases for thicker nanowires.

Copyright line will be provided by the publishe

1 Introduction Metal nanowires (NWs) exhibit various mechanical and electronic properties that cannot be observed on bulk metals [1]. In atom-sized NWs, for example, electrons can pass through NWs without suffering scattering and achieves ballistic conduction even at room temperature. In such ballistic wires, their conductance is determined by the electron transmission probability of NWs and becomes the order of $G_{0}$ where $G_{0} \equiv$ $2 e^{2} / h$ is the quantum unit of conductance. In the ultimate case of single-atom contacts, their conductance is typically $(1-2) G_{0}$ depending on the valency of metal atoms. [1,2].

Another interesting property of NWs is their mechanical behaviors. NWs are virtually free of internal dislocation sources and can achieve a high mechanical strength close to an ideal strength of crystals $[3,4]$. Absence of dislocations sources also makes it difficult for NWs to undergo continuous deformations. As a result, when NWs are elongated, they exhibit discrete necking deformations and often repeat cycles of an elastic stretching and a subsequent abrupt rearrangement of atoms, as reported in simulations [5-8] and experiment [9]. This unique necking deformation of NWs is not only an interesting subject in its own light and but also an important process for fabricating single-atom contacts. In many experiments, single-atom contacts of metals are produced by the so-called break junction method, where a pair of metal electrodes is first brought into contact and then separated apart. The contact point is pulled out and forms a constriction. Further necking deformation of the constriction reduces its size to that of atoms and sometimes yields a single-atom contact. The necking deformation of NWs is thus a crucial process of obtaining single-atom contacts of metals. The necking deformation of NWs is, however, quite different from that of bulk materials as noted above and also varied for different metals. As a result, many problems are left unexplored.

Among metal NWs, those of archetypal FCC metals such as $\mathrm{Au}, \mathrm{Ag}$, and $\mathrm{Cu}$ have been well investigated in the past, and their properties are documented. Break-junction experiments on $\mathrm{Au}$, for example, show that the conductance histogram of breaking $\mathrm{Au}$ junctions exhibits a sharp peak at $1 G_{0}$ both at $300 \mathrm{~K}$ and $4 \mathrm{~K}$ [1]. Because the singleatom conductance of $\mathrm{Au}$ is known to be $1 G_{0}$, this $1 G_{0}$ peak unambiguously corresponds to Au single-atom contacts and proves that the break of $\mathrm{Au}$ junctions can yield single-atom contacts at $300 \mathrm{~K}$ and $4 \mathrm{~K}$. Molecular dynamics (MD) simulations of the break of Au NWs [5-7] show the formation of single-atom contacts and thus support the experimental observations. These findings are understand- 
able considering the high deformability of Au even at low temperatures. Such consistent understanding cannot, however, be extended to other metals, especially for non-FCC metals. A typical example would be the case of $\mathrm{Mg}$. Break junction experiment on $\mathrm{Mg}$ [10] shows that the conductance histogram of $\mathrm{Mg}$ exhibits a sharp peak, presumably the single-atom peak, around $1 G_{0}$ at $4 \mathrm{~K}$. However, at 300 $\mathrm{K}$, the histogram shows no peaks below $4 G_{0}$. Missing of the single-atom peak in a histogram at $300 \mathrm{~K}$ has also been reported in break-junction experiments on $\mathrm{Zn} \mathrm{[11]}$ and $\mathrm{Ga}$ [12]. Clearly, the necking deformation of these nonFCC metal NWs should be different from that of $\mathrm{Au}$ and somehow incapable of forming single-atom contacts at 300 $\mathrm{K}$. In our previous work [13], we carried out break-junction experiments on special $\mathrm{Mg}$ alloys of high ductility and retrieved a couple of low-conductance $\mathrm{Mg}$ peaks that were missing in the conductance histogram of ordinary $\mathrm{Mg}$ at $300 \mathrm{~K}$. This result confirms that the deformation characteristics of $\mathrm{Mg}$ NWs should be a key factor for the formation of $\mathrm{Mg}$ single-atom contacts at $4 \mathrm{~K}$ and their absence $300 \mathrm{~K}$. To obtain some insights on this problem, we have carried out MD simulations of the break of $\mathrm{Mg}$ NWs and studied their necking deformations at $4 \mathrm{~K}$ and at $300 \mathrm{~K}$. MD simulations have been extensively used in the past for examining deformations of various metal NWs. All previous studies have, however, been made on FCC metals and no break simulations on BCC and HCP NWs. Because deformation behaviors of bulk BCC and HCP metals are quite different from those of FCC metals [14], it would be interesting to simulate the necking deformations of HCP Mg NWs and examine how they differ from those of FCC NWs.

2 Computational methods Our NWs consist of a straight pillar of $\mathrm{Mg}$ atoms. We aligned the pillar axis along three low-index directions, [0001], [0110], [1̄120]. Pillars oriented along the $[22 \overline{4} 3]$ direction were also constructed for investigating the slip system as will be discussed in Sec. 3.3. Pillars of different orientations have different cross-sectional shapes reflecting the symmetry of the atomic arrangement. The [0001] pillars, for example, have a hexagonal cross section and are six-sided while the [0110] pillars are a square rod. Each end of the pillar is terminated by a $\mathrm{Mg}$ plate, the shape of which is also hexagonal and square for the [0001] and [0 $\overline{1} 10]$ pillars, respectively. Each pillar consists of 30 atomic layers, and two end plates have 3 atomic layers for each. The length, the number of atoms, and other parameters of the three low-index Mg NWs are tabulated in Table 1. The initial geometry of the $[0001]$ and $[0 \overline{1} 10]$ NWs can be seen at the leftmost figure in Figs. 1(a), (b) and 1(c), (d) respectively.

MD simulations have been carried out at $4 \mathrm{~K}$ and $300 \mathrm{~K}$ employing a simulation software SCIGRESS ME 2.0 (Fujitsu, Ltd.). Throughout all our simulations, the MD time step is $1 \mathrm{fs}$ and the Gear algorithm was used for numerical integrations of the equations. For temperature control, the scaling of atom velocities was made at every MD step.
Table 1 Number of atoms and other parameters of three $\mathrm{Mg}$ NWs tested in our break simulations. $N_{t}$ and $N_{a}$ represent the total number of atoms in a NW and the number of atoms in the pillar part, respectively, and $h$ measures the initial height of the pillar part.

\begin{tabular}{rrrr}
\hline & {$[0001] \mathrm{NW}$} & {$[0 \overline{1} 10] \mathrm{NW}$} & {$[\overline{1} \overline{1} 20] \mathrm{NW}$} \\
\hline Cross section & hexagonal & square & rectangular \\
$N_{t}$ & 990 & 912 & 927 \\
$N_{a}$ & 540 & 570 & 435 \\
$h(\mathrm{~nm})$ & 5.436 & 5.402 & 4.447 \\
\hline
\end{tabular}

A tight-binding type EAM potential [15] was used for the interatomic potential for $\mathrm{Mg}$. First, atoms in the pillar part are relaxed. Then, the pillar is stretched by moving the top and bottom plates in the opposite directions at a constant velocity of $2 \mathrm{~m} / \mathrm{s}$. Atoms in the two plates are frozen during relaxing and stretching the pillar. We note that the atomic arrangement of real break junctions must be far less perfect than that of NWs shown in Fig. 1. Probably because of this inevitable disorder in their atomic configurations, the break of real junctions becomes varied and irreproducible and should be treated as stochastic processes. To take into account this stochastic nature of the junction break, we followed the statistical scheme employed in the break simulations of Ni NWs $[16,17]$ and repeated the break simulation one hundred times with each time randomly changing the initial velocity distribution of atoms.

Two quantities are evaluated in each break simulation in addition to the snapshots of breaking NWs. One is the stretching force which is computed at every MD steps following the procedures described by Finbow et al. [18]. Another quantity is the minimum cross section $S_{m}$ of an NW which represents the cross-sectional area of the narrowest constriction of the NW. This parameter, in the form of the smallest constriction radius, has been first discussed by Bratkovsky et al. [5] and subsequently employed in many MD simulations of NWs for studying their neck evolution. In this work, we calculated $S_{m}$ in the following manner. First, we cut an NW perpendicular to its axis into slices whose thickness is equal to the interplanar distance between two adjacent close-packed planes of Mg. Next, the volume of each slice $V_{\text {slice }}$ is calculated assuming that each atom is a sphere occupying an atomic volume defined as $V_{\text {atom }}=$ (the volume of the HCP unit cell)/(the number of atoms in the unit cell). Then, the smallest $V_{\text {slice }} / V_{\text {atom }}$ among the slices defines the minimum cross section $S_{m}$. As can be understood from this definition, $S_{m}$ essentially represents the number of atoms locating at the narrowest constriction. We computed $S_{m}$ at every 2000 or 3000 MD steps and investigated how $S_{m}$ evolves with the NW stretching.

\section{Results and discussion}

3.1 Deformation morphology of $\mathbf{M g ~ N W s ~ W e ~}$ show in Fig. 1 the necking evolution of Mg NWs un- 
der stretching. Panels (a) and (b) represent snapshots of [0001]-NWs at $4 \mathrm{~K}$ and $300 \mathrm{~K}$, respectively, and panels (c) and (d) are for [0110]-NWs. It can be clearly seen in all panels that NWs undergo necking and break nearly at the midpoint of the pillar. This guarantees that no stress concentration occurs at the pillar-plate junctions. For the [0001]-NW at $4 \mathrm{~K}$ shown in Fig. 1(a), necking proceeds locally and produces a cone-shaped constriction. On the other hand, necking deformation of other NWs leads to a formation of a thinner NW before breakup. In Fig. 1(c), the thinner NW makes further necking and produces a singleatom contact. At $300 \mathrm{~K}$, however, Mg NWs exhibit break behaviors which are quite different from those at $4 \mathrm{~K}$. The [0001]-NW shown in Fig. 1(b) undergoes a premature break before shrinking to the size of one or two atoms. On the other hand, stretching of [0110]-NWs at $300 \mathrm{~K}$ often yields a very long atomic wire as seen in the third snapshot in Fig. 1(d). This wire shows a unique atomic geometry and will be discussed separately in Sec. 3.4.

To elucidate the neck evolution more quantitatively, we calculated for each NW the minimum cross section $S_{m}$ mentioned in Sec. 2 and examined its temporal variation. Figures 2(a) and (b) show examples of the $S_{m}$ trace obtained on [0001]- and [0110]-NWs, respectively. In each figure, $S_{m}$ is plotted as a function of the NW elongation, and the blue and the red lines represent the variation of $S_{m}$ at $4 \mathrm{~K}$ and $300 \mathrm{~K}$, respectively. As reported in previous simulations of FCC NWs [5-8,16], $S_{m}$ traces at 4 K decrease stepwise and exhibit plateaus and abrupt steps between them. Such step structure is clearly visible in Fig. 2(a) for [0001] NW, where well-defined steps continue to appear down to the final breakup. On the other hand, the $S_{m}$ trace of the $[0 \overline{1} 10] \mathrm{NW}$ at $4 \mathrm{~K}$, shown in Fig. 2(b), exhibits step structures at the initial and the final deformation stages but varies nearly continuously for $S_{m} \sim(5-15)$. On the other hand, the stepwise behavior becomes unclear for the $S_{m}$ traces at $300 \mathrm{~K}$. In the $S_{m}$ trace of [0001] NW shown in Fig. 2(a), steps are still visible in the middle of stretching but unrecognizable for $S_{m}<5$. Quite different is the $S_{m}$ trace of the $[0 \overline{1} 10] \mathrm{NW}$ at $300 \mathrm{~K}$ shown in Fig. 2(b) where the trace exhibits a long plateau at $S_{m}=5$. This plateau corresponds to the formation of the long atomic wire seen in Fig. 1(d), and the plateau at $S_{m}=5$ indicates that the atomic wire is five-atom thick. Details of this wire will be discussed in Sec. 3.4.

Along with [0001] and [0110] NWs, we carried out

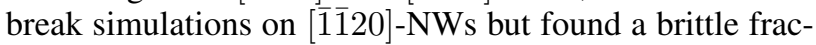
ture both at $4 \mathrm{~K}$ and at $300 \mathrm{~K}$. One $[\overline{1} 120]-\mathrm{NW}$ at $4 \mathrm{~K}$ shows a $S_{m}$ trace that exhibits a long plateau around $S_{m} \sim 17$ and then jumps to zero. Similar brittle behaviors are observed for other [1 $1 \overline{1} 20]-N W s$ at $4 \mathrm{~K}$ and also at $300 \mathrm{~K}$. Clearly [11120]-NWs tend to make a premature break well before they become the size of atoms and would make no substantial contributions to the formation of $\mathrm{Mg}$ single-atom contacts. We will, therefore, make no further discussion on
[1]120]-NWs and henceforth focus our attention to [0001] and [01ㅣㄹ NWs.

As mentioned in Sec. 2, we emulated the real junction break by carrying out many simulations with different initial velocity distributions. In every simulation, a NW follows a different deformation path and yields a different $S_{m}$ trace. We accumulated one hundred $S_{m}$ traces and constructed the histogram of $S_{m}$. The results are shown in Figs. 3(a) and (b) for [0001] and [0110] NWs, respectively. As in Fig. 2, the blue and the red lines represent the results obtained at $4 \mathrm{~K}$ and $300 \mathrm{~K}$, respectively.

In Fig. 3(a), the $S_{m}$ histogram of [0001] NWs at $4 \mathrm{~K}$ exhibits well-defined peaks located at $S_{m}=1,2,3, \cdots$. Appearance of these integer peaks indicates that a constriction containing $n=1,2,3, \cdots$ atoms should be stable and more preferentially formed than other geometries. These integer peaks in the histogram also correspond to the integer steps in the $S_{m}$ trace shown in Fig. 2(a). These results suggest that [0001] NWs at $4 \mathrm{~K}$ undergo discontinuous necking and discretely reduce their constriction size atomby-atom. We note that similar integer peaks have been observed in the $S_{m}$ histogram of other metals [7,8,16,19]. Among integer peaks shown in Fig. 3(a), the single-atom peak at $S_{m}=1$ has the highest intensity. On the other hand, the peak structure is less clear in the $S_{m}$ histogram of [0110]-NWs at $4 \mathrm{~K}$ shown in Fig. 3(b). This means that [0110] NWs have no preferred atomic geometry for their constrictions. Nevertheless, the single-atom peak still appears as a main peak. These results on two NWs suggest that the single-atom contacts of $\mathrm{Mg}$ would always be a preferred geometry at $4 \mathrm{~K}$.

The $S_{m}$ histogram significantly changes when the temperature rises to $300 \mathrm{~K}$. In the histogram of [0001] NWs shown in Fig. 3(c), the single-atom peak at $S_{m}=1$ is still visible but other integer peaks are nearly buried in the increased background, except for the enhanced peaks at $S_{m} \sim 2.5$ and $S_{m}=5$. The intensity of the singleatom peak is lower than that at $4 \mathrm{~K}$ and nearly half of that of the $S_{m}=5$ peak. The same peak enhancement at $S_{m} \sim 2.5$ and $S_{m}=5$ occurs more dramatically in the histogram of $[0 \overline{1} 10]$ NWs shown in Fig. 3(d) where peaks other than the $S_{m} \sim 2.5$ and $S_{m}=5$ peaks almost disappear. Clearly, the large $S_{m}=5$ peak corresponds to the long plateau and the long atomic wire shown in Fig. 2(b) and Fig. 1(d), respectively. As seen in Fig. 2(b), the long atomic wire with $S_{m}=5$ shrinks to a thinner wire with $S_{m} \sim 2.5$ and then snaps off without further thinning down to a single-atom contact. Similar break can be observed for other $S_{m}=5$ wires. This rather brittle behavior of the $S_{m}=5$ and $S_{m} \sim 2.5$ wires explains why their preferred formation at $300 \mathrm{~K}$ relatively suppresses the formation of single-atom contacts. On the other hand, NWs at $4 \mathrm{~K}$ show no such strong preference and can be reduced to singleatom contacts. It is not clear at this time why $S_{m} \sim 2.5$ and $S_{m}=5$ wires are abundantly formed at $300 \mathrm{~K}$ but not 
(a) $[0001] \mathrm{NW} \quad 4 \mathrm{~K}$

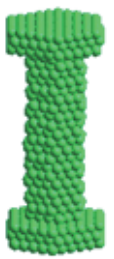

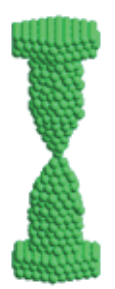

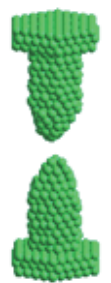

(c) $[0 \overline{1} 10] \mathrm{NW} \quad 4 \mathrm{~K}$
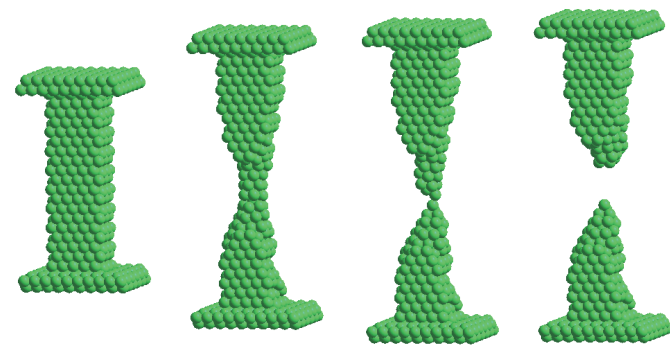

(b) $[0001] \mathrm{NW} 300 \mathrm{~K}$

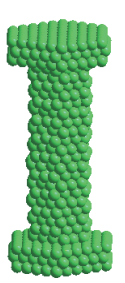

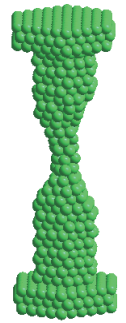

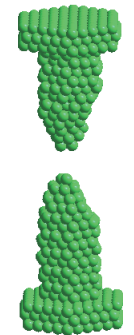

Figure 1 Necking evolution of Mg NWs under stretching; (a) [0001]-NW at $4 \mathrm{~K}$, (b) [0001]-NW at $300 \mathrm{~K}$, (c) [0110]-NW at $4 \mathrm{~K}$, (d) $[0 \overline{1} 10]-\mathrm{NW}$ at $300 \mathrm{~K}$.

(a) $[0001] \mathrm{NW}$

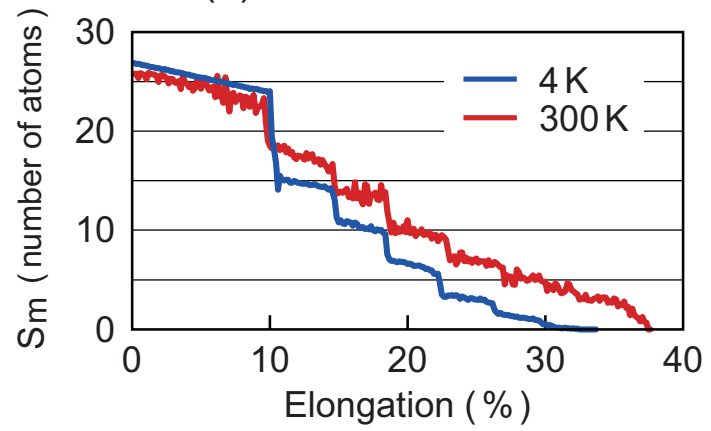

(b) $[0 \overline{1} 10] \mathrm{NW}$

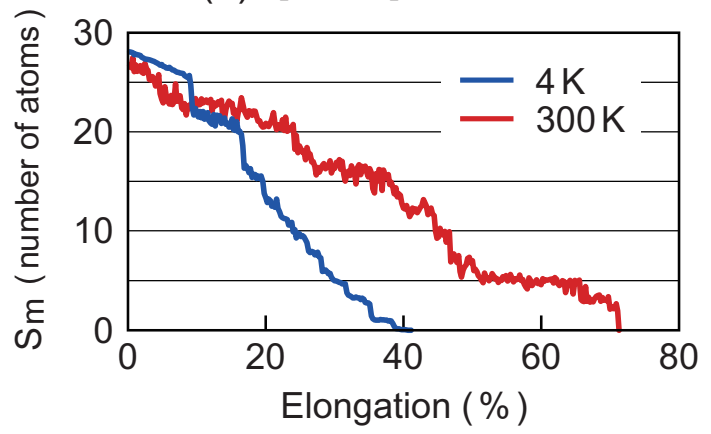

Figure 2 Temporal variation of the minimum cross section $S_{m}$ for (a) [0001]-NW at 4 K (blue) and $300 \mathrm{~K}$ (red) and (b) [0110]-NW at $4 \mathrm{~K}$ (blue) and $300 \mathrm{~K}$ (red).

at $4 \mathrm{~K}$. Some discussion on this point will be made for the $S_{m}=5$ wires in Sec. 3.4.

We now compare our $S_{m}$ histograms with the experimental conductance histograms of $\mathrm{Mg}[10,13]$. Comparison of $S_{m}$ and conductance histograms is not generally straightforward because $S_{m}$ peaks do not necessarily correspond to peaks in the conductance histogram: NWs of different $S_{m}$ sometimes yield nearly the same conductance and contribute to the same conductance peak. An exception would be the case for the first (the lowest-conductance) peak in a conductance histogram. Conductance calculations $[7,8]$ show that the first conductance peak mainly comes from the contributions of $S_{m}=1 \mathrm{NWs}$, and this is particularly true for $s$-electron metals such as $\mathrm{Au}$ whose $1 G_{0}$ peak can be safely identified as the single-atom peak. Because $\mathrm{Mg}$ also has $s$-like valence electrons, it can be expected that the $1 G_{0}$ peak of $\mathrm{Mg}$ observed by Smit et al. [10] would correspond to the $S_{m}=1$ peak in the $S_{m}$ histogram. 

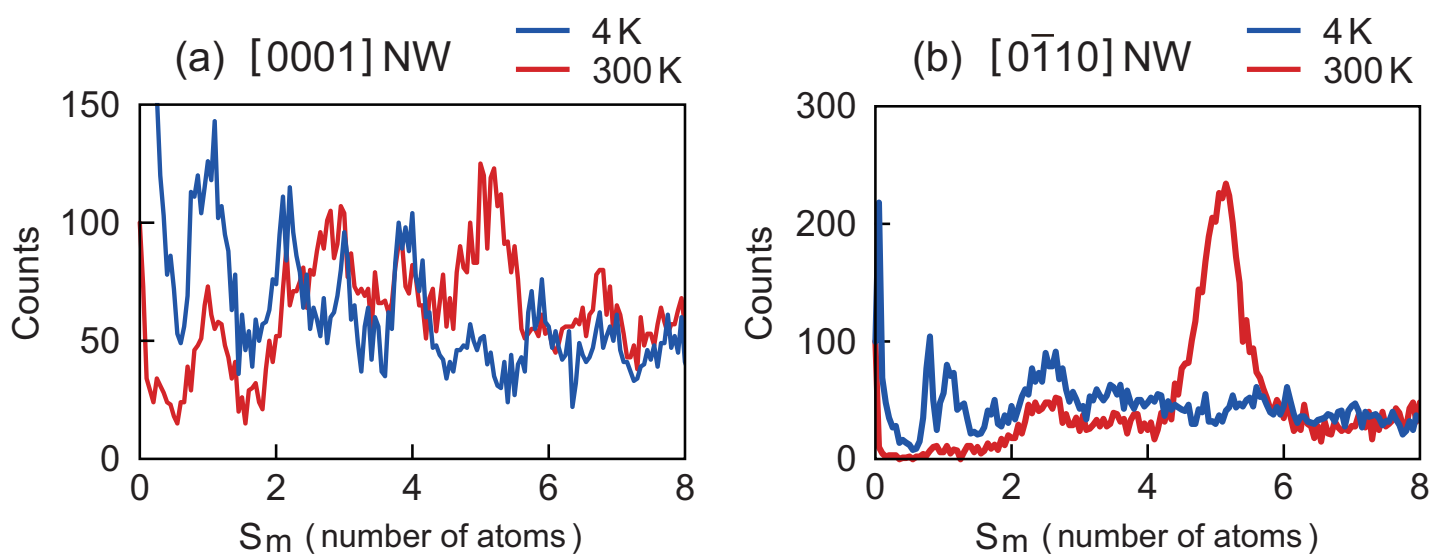

Figure 3 Histogram of $S_{m}$ constructed from $100 S_{m}$ traces. Panels (a) and (b) show the $S_{m}$ histogram for [0001] and [0110] NWs, respectively. As in Fig. 2, the blue and the red lines represent the histogram obtained at $4 \mathrm{~K}$ and $300 \mathrm{~K}$, respectively.

Then, our simulation results on the $S_{m}=1$ peak, i.e. it is a dominant peak at $4 \mathrm{~K}$ but becomes suppressed at $300 \mathrm{~K}$, consistently agree with the experimental observations that the $1 G_{0}$ peak appears in the conductance histogram at $4 \mathrm{~K}$ but disappears at $300 \mathrm{~K}[10,13]$.

3.2 Stretching force During NW stretching, we computed the force at every MD steps as mentioned in Sec. 2. Figures 4(a) and (b) depict the stretching force as a function of the NW elongation measured in $\%$ for [0001]and $[0 \overline{1} 10]-N W s$, respectively. At $4 \mathrm{~K}$, the force exhibits a sawtooth behavior: it first increases linearly with the elongation and then sharply decreases. Similar sawtooth behavior has been commonly observed in previous break simulations [5-8] and experiments [3,4,9] on other metals and interpreted as a repetition of elastic deformation and subsequent rapid plastic deformation, or an atomic rearrangement. On the other hand, the necking deformation of Mg NWs becomes less discrete at $300 \mathrm{~K}$. For the [0001] NW at $300 \mathrm{~K}$ shown in Fig.4(a), the sawtooth pattern can be observed up to around 40\%-elongation but becomes unclear near the breakup. Effects of thermal smearing are more evident for $[0 \overline{1} 10]$ NWs. In Fig.4(b), the stretching force at $300 \mathrm{~K}$ shows the sawtooth behavior only at the first stage but varies nearly continuously for the rest of stretching.

Thermal effects are also observed on the magnitude of the yield force of Mg NWs. By comparing the forceelongation curves shown in Figs. 4(a) and (b), we can find that the force at the first sawtooth peak, i.e. the yield force, is significantly higher at $4 \mathrm{~K}$ than at $300 \mathrm{~K}$. The second peak force is also higher at $4 \mathrm{~K}$, particularly for the $[0 \overline{1} 10]$ NW. These results agree with the fact that yield stress of many metals increases with lowering temperature. With the progress of necking deformation, however, the difference between the stretching force at $4 \mathrm{~K}$ and that at $300 \mathrm{~K}$ decreases and, at the later part of the force-elongation curve, the force at $4 \mathrm{~K}$ tends to be lower than that at $300 \mathrm{~K}$. This result indicates that, when an $\mathrm{Mg} \mathrm{NW}$ shrinks to the size of a few atoms, its deformation force becomes insensitive to temperature, and the final deformations near the breakup develop nearly athermally. If the same situation holds for metals other than $\mathrm{Mg}$, it might explain an apparently puzzling observation that the break of some hard metals can produce single-atom contacts even at $4 \mathrm{~K}$ though they show very high yield stress at cryogenic temperatures. The tensile stretching of such hard NWs at $4 \mathrm{~K}$ would initially needs a large force but, after their neck being reduced to the size of atoms, their later deformations proceed under the same force level as that required at $300 \mathrm{~K}$.

In Fig. 4(a), the last plateau of the stretching force at $4 \mathrm{~K}$, appearing just before the NW breakup, represents the break force of the single atom contact of $\mathrm{Mg}$ at $4 \mathrm{~K}$. For the [0001] NW shown in the figure, the break force is $\sim 0.25$ $\mathrm{nN}$. Other force curves yield similar values for the singleatom break force of $\mathrm{Mg}$. This value can be compared with that of other metals. Previous break simulations [8] show that the single-atom break force of $\mathrm{Au}, \mathrm{Ag}, \mathrm{Pt}$, and $\mathrm{Ni}$ is $0.81 \mathrm{nN}, 0.60 \mathrm{nN}, 1.12 \mathrm{nN}$, and $1.66 \mathrm{nN}$, respectively. Experimentally, the break force of the Au single atom contact has been measured to $1.5 \mathrm{nN}$ at $4 \mathrm{~K}$ [9]. Compared to these theoretical and experimental valuesCthe break force of $\mathrm{Mg}$ single-atom contacts is significantly lower and amounts to only $15 \%$ of the experimental break force of Au singleatom contacts. The low break force of $\mathrm{Mg}$ would not, however, be unexpected because $\mathrm{Mg}$ is a soft metal and has a low cohesive energy that is only $40 \%$ of that of Au. At 300 $\mathrm{K}$, experiments $[3,4]$ on $\mathrm{Au}$ and $\mathrm{Pt}$ find that the single-atom contacts of these metals rupture at $1.6 \mathrm{nN}$ and $0.6 \mathrm{nN}$, respectively. The force curves at $300 \mathrm{~K}$, however, show large fluctuations near the breakup, and it is virtually impossible to accurately determine the single-atom break force of $\mathrm{Mg}$ 
(a) $[0001] \mathrm{NW}$

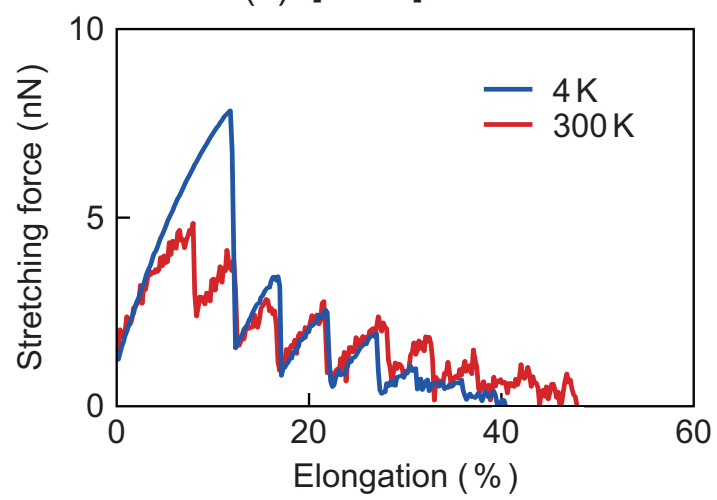

(b) $[0 \overline{1} 10] \mathrm{NW}$

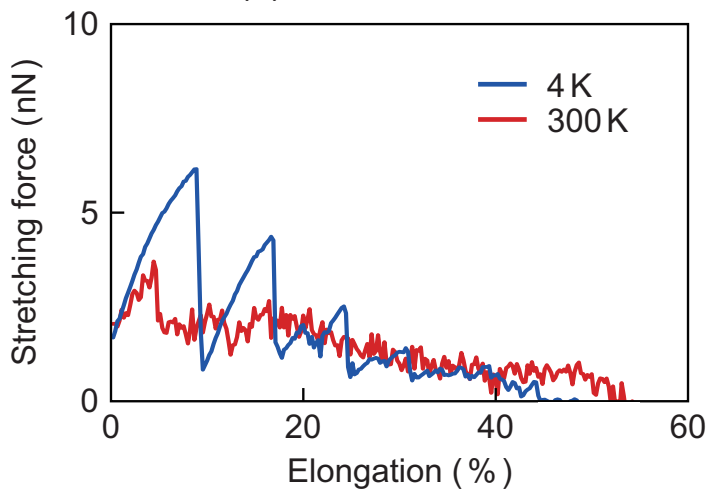

Figure 4 Temporal variation of the stretching force for (a) [0001]-NW at $4 \mathrm{~K}$ (blue) and $300 \mathrm{~K}$ (red) and (b) [0110]-NW at $4 \mathrm{~K}$ (blue) and $300 \mathrm{~K}$ (red).

at $300 \mathrm{~K}$ and compare it with experimental values of other metals.

3.3 Basal plane slip Unlike FCC metals, HCP metals have a limited number of active slip systems, and plastic deformations of HCP metals proceed almost exclusively through the basal plane slip (and twinning) [14]. Activation of non-basal slips requires high energies and is usually realized only at large stresses and high temperatures. In our stretching of [0001]- and [0110]-NWs, however, the basal plane has a null Schmidt factor. This might be the reason why these NWs at $4 \mathrm{~K}$ show high yield strength in Fig. 3. In the case of the [0110]-NWs, subsequent necking deformations appear to proceed through slip deformations along prismatic planes as seen in Fig. 1(c).

To realize a non-zero Schmidt factor for the basal plane, we prepared NWs oriented along the [22 $\overline{4} 3]$ direction and stretched them at $4 \mathrm{~K}$. The results are shown in Fig. 5. As seen in the figure, the first slip occurs along the basal plane. During subsequent deformations, the basal plane slip often takes place and, along with other deformation systems, contributes to the neck evolution. In the case shown in Fig. 5, the basal plane slip occurs near the NW breakup and yields a single-atom contact. The overall deformation behavior of the $[22 \overline{4} 3] \mathrm{NW}$ is not much different from those of [0001] and [0110] NWs. This suggests that the Schmidt factor for the basal plane slip would have little influence on overall necking evolution of NWs, and our results obtained on [0001] and [0110] NWs at $4 \mathrm{~K}$ would represent the typical deformation behavior of Mg NWs.

\subsection{Icosahedral nanowires}

3.4.1 Formation of icosahedral nanowires We now discuss in some detail the long atomic wire shown in the snapshot in Fig. 1(d). This atomic wire is also responsible for the prolonged plateau in Fig. 2(b) and the huge $S_{m}=5$ peak in Fig. 3(b). Figure 6(a) depicts an atomic structure of one such long atomic wire formed by stretching a $[0 \overline{1} 10]-N W$. Arrangement of atoms viewed along the wire axis is illustrated in Fig. 6(b). The wire shows a pentagonal cross section and clearly takes an atomic geometry different from that of ordinary HCP crystals. In Fig. 6(b), the top atom connects to five atoms, colored blue, in the second layer. These five atoms in the second layer form a pentagonal ring and bond to a single center atom in the third layer which in turn links to five atoms consisting the forth layer. Thus, the wire has a $1-5-1-5-\cdots$ stacking. The 1-5-1-5-1 unit forms an icosahedron with one atom at its center. The atomic arrangement of the wire can therefore be viewed as a sequence of interpenetrated icosahedra. Similar icosahedral or pentagonal nanowires have been observed in previous break simulations of BCC $\mathrm{Na}$ [20] and some FCC metals such as Ni [16,17,21-24], $\mathrm{Cu}[24,25], \mathrm{Au}$ [26], and $\mathrm{Al}$ [24]. Our results show that the formation of icosahedral NWs is not a special event limited to BCC and FCC metals but should be a more robust phenomenon observable for other metals including HCP $\mathrm{Mg}$.

Comparison of snapshots in Figs. 1(b) and (d) indicates that the stretching of [0110]-NWs tends to produce longer icosahedral NWs than in the case of [0001]-NWs. To examine this difference more quantitatively, we calculated the lifetime $\tau$ of an icosahedral NW and compared the distribution of $\tau$ for two NWs. Because we stretch NWs at a constant rate, $\tau$ would yield a measure of the icosahedral NW length if we assume that the total NW elongation is entirely due to the growth of the icosahedral part. The lifetime of an icosahedral NW can be obtained by measuring the length of the $S_{m}=5$ plateau in the $S_{m}$ trace. We first defined the $S_{m}=5$ plateau as the one which lies in the range $4.8 \leq S_{m} \leq 5.3$ and, for each $S_{m}$ trace at 300 $\mathrm{K}$, measured the length of such $S_{m}=5$ plateau. The resulting distribution of $\tau$ is plotted in Fig. 7 for [0001] and [0110]-NWs. In Fig. 7, the data points at $\tau=0$ ps represents the fraction of those $S_{m}$ traces that show no $S_{m}=5$ plateaus or marginal ones with $\tau<40 \mathrm{ps}$. In the case of 


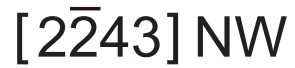
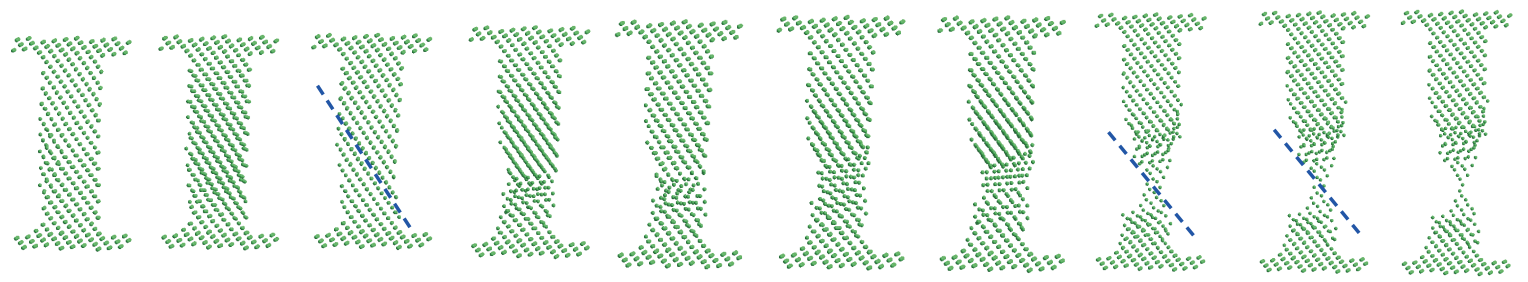

Figure 5 Necking evolution of a [22 $\overline{4} 3]$ NW under stretching. Dashed lines indicate the occurrence of the basal plane slip.

(a)

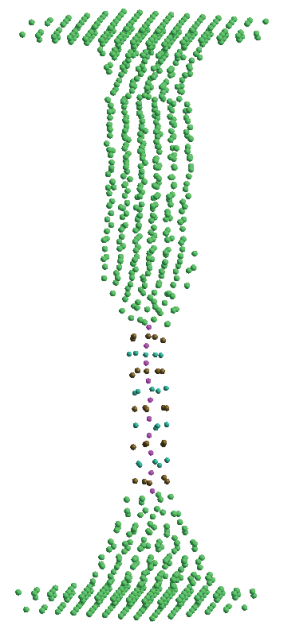

(b)

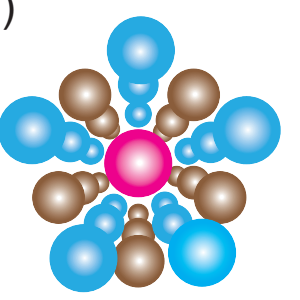

Figure 6 (a) Atomic structure of an icosahedral NW of Mg formed by stretching a [0110]-NW at $300 \mathrm{~K}$ (b) Arrangement of atoms viewed along the wires axis.

[0001] NWs, this fraction is $52 \%$, i.e. more than half of the [0001]-NW produce no well-defined icosahedral nanowires at their break. On the other hand, the fraction decreases to $25 \%$ for [0110]-NWs. Also in the long $\tau$ side, the distribution for [0110]-NWs extends up to 400 ps whereas the distribution rapidly drops for [0001] NWs at $\tau>40 \mathrm{ps}$. These findings clearly indicate that the formation of long icosahedral NWs depends on the NW orientation and is more favored for [0110]-NWs than [0001] NWs.

Icosahedral $\mathrm{NWs}$ of $\mathrm{Ni}, \mathrm{Cu}$ and $\mathrm{Al}$ have been investigated in detail by García-Mochales and coworkers [16, 17,21-24]. They showed that the formation of icosahedral NWs is temperature dependent and most favored at $T \sim(0.2-0.3) T_{m}$ where $T_{m}$ is the melting temperature. In break simulations of Ni NWs at $4 \mathrm{~K}$, icosahedral NWs are rarely observed. These results on Ni icosahedral NWs are in good agreement with our results $\mathrm{Mg}$ icosahedral NWs, the formation of which is also enhanced at $300 \mathrm{~K}$ but suppressed at $4 \mathrm{~K}$. García-Mochales and coworkers also reported that [100] and [110] NWs show higher productiv-

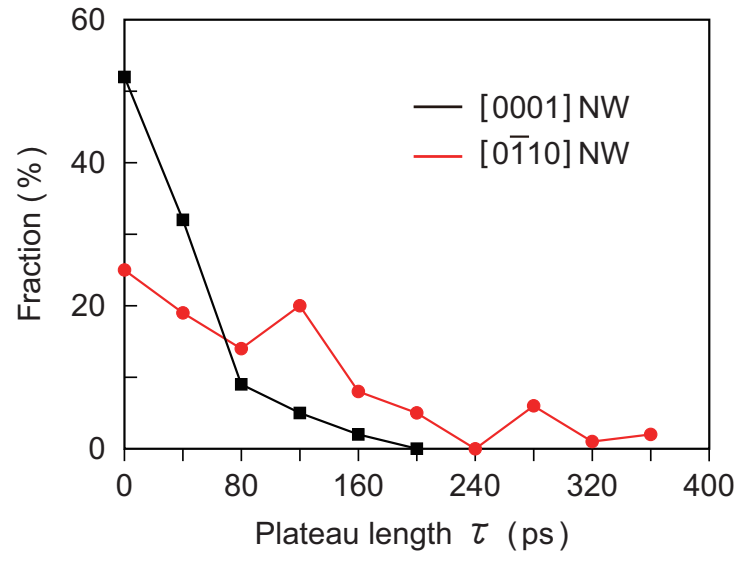

Figure 7 Distribution of the plateau length (or lifetime) of the $S_{m}=5$ plateau obtained for [0001]- (black) and [0110] (red) NWs at $300 \mathrm{~K}$. The bin width is $40 \mathrm{ps}$, and all data points are plotted at the lowest end of each bin; a data point at $160 \mathrm{ps,} \mathrm{for} \mathrm{example,} \mathrm{indicates} \mathrm{the} \mathrm{fraction} \mathrm{of} \mathrm{those}$ $S_{m}=5$ plateaus that show $160 \leq \tau<200$ ps.

ity of icosahedral NWs than [111] NWs. This orientation dependence is again consistent with the observed low productivity for $\mathrm{Mg}$ [0001] NWs because both the [111] plane of $\mathrm{Ni}$ and the [0001] plane of $\mathrm{Mg}$ are a close-packed plane and have the same atomic arrangement.

Long icosahedral NWs indicate their superior stability. In fact, theoretical calculations [27-29] have already demonstrated that they are indeed energetically favorable compared to other geometries. We here point out that the stability of icosahedral NWs can also be intuitively understood from their atomic arrangement. In the $1-5-$ $1-5-\cdots$ stacking of icosahedral NWs, the center atom on the NW axis has twelve neighbors. This coordination number is the same as that of FCC and HCP crystals. The local packing density is thus nearly the same as that of those close packed structures. It is also mathematically proven [30] that the local FCC and HCP stacking of atoms, i.e. one center atom surrounded by twelve nearest neighbor atoms, can continuously transform to the $1-5-1-5-\cdots$ 
stacking without changing the bond length between the center atom and the surrounding atoms. This suggests that the FCC/HCP $\leftrightarrow$ icosahedral transformation would proceed with a low energy barrier. It is then quite conceivable that once a small icosahedral "nucleus" or a "core" is formed at the neck of an NW, the nucleus can easily grows into a long icosahedral wire with stretching the NW. According to this interpretation, the formation of icosahedral NW depends on whether such an icosahedral core is created at the NW neck. The lower chance of obtaining icosahedral NWs for [0001] NWs would suggest that such nuclei are rarely produced for the [0001] stacking. We also consider that nucleation of the icosahedral core would be more favorable at higher temperatures. This might explain why icosahedral NWs are abundantly observed at $300 \mathrm{~K}$ but not at $4 \mathrm{~K}$.

3.4.2 Comparison with experiment In view of their high stability and abundance, it seems puzzling that the icosahedral NWs appear to make no contributions to the experimental conductance histograms. In previous experiment [10], the conductance histogram of $\mathrm{Mg}$ at $300 \mathrm{~K}$ shows multiple peaks but these peaks are interpreted as due to the shell structure of Mg NWs. Another experiment [13] on $\mathrm{Mg}$ reports a broad maximum around $5 G_{0}$ in the conductance histogram at $300 \mathrm{~K}$. The observed peak, however, looks different from the sharp $S_{m}=5$ peak shown in Fig. 3(b). Similarly for Ni NWs, the break simulations exhibit the formation of icosahedral NWs and predict a well-defined $S_{m}=5$ peak in the $S_{m}$ histogram. However, the experimental conductance histogram of $\mathrm{Ni}$ at 300 $\mathrm{K}$ [31] shows no peak features attributable to icosahedral NWs. The only direct evidence obtained so far on icosahedral NWs is a transmission-electron-microscopy observation [32] of a pentagonal structure of $\mathrm{Cu}$. This is, however, a small structure consisting of a few atoms and quite different from a long wire shown in Fig. 6(a). It might be possible that the sharp $S_{m}=5$ peak does not produce a corresponding peak in the conductance histogram, particularly when the conductance of icosahedral NWs shows a broad distribution and only produces a smooth feature in the histogram. At this time, we have no information on the conductance of $\mathrm{Mg}$ icosahedral NWs but, considering the structural robustness of the $1-5-1-5-\cdots$ stacking, large fluctuations of the conductance appear unlikely.

We consider that icosahedral NWs provide little experimental evidences because they are rarely formed in real break junctions at $300 \mathrm{~K}$. As mentioned before, the formation of a long icosahedral $\mathrm{NW}$ would require a core or a nucleus from which the $1-5-1-5-\cdots$ structure grows. In the break of real junctions, their neck evolves along a wide variety of deformation paths, and there might be a slim chance for the neck to follow the right deformation path that leads to the nucleation of an icosahedral core. In simulations, on the other hand, NWs are initially a perfect crystal and stay less disordered than real junctions during break processes. As a result, the variety of the de-

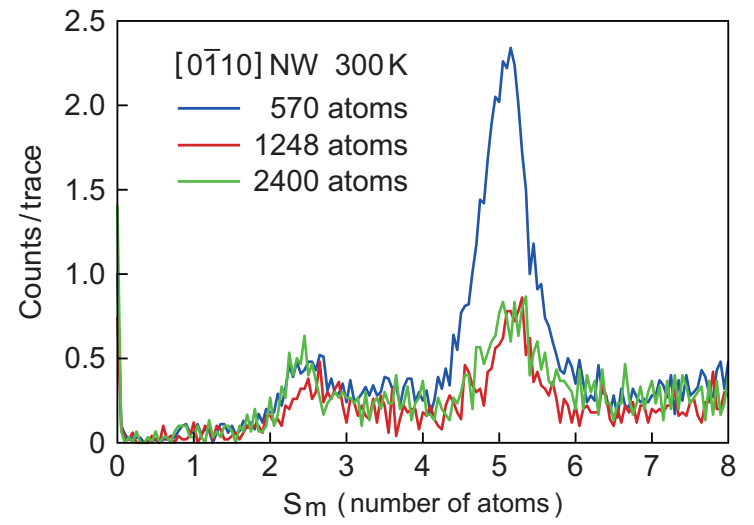

Figure 8 Histogram of $S_{m}$ obtained at $300 \mathrm{~K}$ for three [0110] NWs of different size. Blue, red and green lines are for 570-, 1,248- and 2,400-atom NWs, respectively,

formation paths would be much smaller than for real junctions, and the chance would be higher for the nucleation of icosahedral cores. As mentioned in Sec. 2, we introduced stochasticity in our simulations by randomly changing the initial velocity distribution of atoms, but this may still be insufficient to fully emulate the variety of atomic configurations in real break junctions. To get some insight into this issue, we increased the size of NWs and investigated how the $S_{m}$ histogram changes with the NW size. For larger NWs, their necking deformations would be more varied than for smaller NWs and thus be more closely represent the deformations of real junctions. We prepared two larger [0110] NWs. One of them has a pillar part which consists of 48 atomic layers each containing 26 atoms. The pillar has 1,248 atoms in total and is $6.778 \mathrm{~nm}$ in height. The pillar part of another NW has 2,400 atoms (80 layers of 30 atoms) and $8.542 \mathrm{~nm}$ in height. These two NWs have the same aspect ratio with the one shown in Figs. 1(c), and (d). We carried out 50 and 30 break simulations at $300 \mathrm{~K}$ for the 1,248- and 2,400-atom NWs, respectively, and constructed the $S_{m}$ histogram. The results are shown in Fig. 8 together with our previous result obtained on the 570-atom NWs. The figure clearly shows that the $S_{m}=5$ peak becomes significantly reduced when the number of pillar atoms increases from 570 to 1,248 and 2,400. We also found that the lifetime of icosahedral nanowires becomes shortened for larger NWs: the longest lifetime observed for the 1,248and 2,400-atom NWs are $\sim 240$ ps, which is only half of that for the 570-atom NWs (see Fig. 7). The results clearly indicate that the formation of icosahedral NW becomes less favorable for larger NWs. This size effect thus supports our assumption that large variations in the NW deformation path tend to diminish the chance of forming icosahedral NWs and this might be reason why long icosahedral NWs rarely appear in real break junctions. We note in Fig. 8 that the $S_{m}=5$ peak remains the same when the number of pillar atoms is nearly doubled from 1,248 to 2,400 . 
For the 2,400-atom NWs, stochasticity introduced in our simulations might be still too weak to fully reproduce the variety of atomic configuration in real junctions. We add to note that García-Mochales and coworkers [24] investigated the size dependence of the icosahedral NW formation for FCC metals and found that the formation is less favored for larger NWs. They also pointed out that the fine control over the initial nanocontact cross-section would be critical for experimentally producing long icosahedral NWs. Their results are thus in good agreement with ours on $\mathrm{Mg}$ and also in line with our assumption on the importance of icosahedral core structures for the growth of icosahedral NWs.

4 Summary In the present work, we have studied the tensile break of magnesium NWs using MD simulations and obtained the following results on the necking deformations of [0001] and [0110]-NWs.

First, we found marked differences between their deformation behaviors at $4 \mathrm{~K}$ and those at $300 \mathrm{~K}$. At $4 \mathrm{~K}$, the necking proceeds discretely and leads to the formation of single-atom contacts before NW failure. Correspondingly, the histogram of the minimum cross section $S_{m}$ at $4 \mathrm{~K}$ shows a sharp single-atom peak at $S_{m}=1$. At $300 \mathrm{~K}$, on the other hand, the NW stretching yields the formation and growth of icosahedral NWs at the nanowire neck. In the $S_{m}$ histogram, the $S_{m}=1$ peak becomes suppressed and a large peak appears around $S_{m}=5$. The snapshots show the formation and growth of icosahedral NWs at the nanowire neck. The observed temperature dependence of the $S_{m}$ histogram consistently corresponds to that of the experimental conductance histogram of $\mathrm{Mg}[10,13]$.

The stretching force at $4 \mathrm{~K}$ shows a sawtooth pattern and takes high values in the initial stages of necking deformation. At later stages, however, the force tends to vary continuously and shows nearly the same magnitude at $4 \mathrm{~K}$ and at $300 \mathrm{~K}$. The deformation of $\mathrm{Mg}$ NWs thus becomes almost temperature independent when the NW size is reduced to that of atoms.

The icosahedral NWs observed in our simulations shows a characteristic $1-5-1-5-\cdots$ stacking and has the same atomic structure as icosahedral NWs reported in break simulations of other metals [16, 17, 21-23, 25,26]. We found that the formation of long icosahedral NWs depends on the NW orientation and temperature and is particularly favored for [0110]-NWs and at $300 \mathrm{~K}$. Break simulations carried out on larger NWs containing larger number of atoms, however, showed a smaller chance of obtaining icosahedral NWs. We consider that the growth of long icosahedral NWs requires the formation of a nucleus at the neck. Such a core would have a slim chance to be formed during the break of larger NWs, which involves larger varieties of neck configurations and deformation paths. This might also explain why icosahedral NWs are rarely observed in real break junctions.

\section{References}

[1] N. Agraït, A. Levy Yeyati, and J. M. van Ruitenbeek, Phys. Rep. 377, (2003) 81.

[2] J. C. Cuevas, A. Levy Yeyati, and A. Martín-Rodero, Phys. Rev. Lett. 80, (1998) 1066.

[3] T. Kizuka, Phys. Rev. B 77, (2008) 155401.

[4] T. Kizuka and K. Monna, Phys. Rev. B 80, (2009) 205406.

[5] A.M. Bratkovsky, A. P. Sutton, and T. N. Todorov, Phys. Rev. B 52, (1995) 2036.

[6] M.R.Sörensen, M. Brandbyge, and K. W. Jacobsen, Phys. Rev. B 57, (1998).

[7] M. Dreher, F. Pauly, J. Heurich, J.C. Cuevas, E. Scheer, and P. Nielaba, Phys. Rev. B 72, (2005) 075435.

[8] F. Pauly, M. Dreher, J. K. Viljas, M. Häfner, J. C. Cuevas, and P. Nielaba, Phys. Rev. B 74, (2006) 235106.

[9] G. Rubio, N. Agraït, and S. Vieira, Phys. Rev. Lett. 76, (1996) 2302.

[10] R. H. M. Smit, A. I. Mares, M. Häfner, P. Pou, J. C. Cuevas, and J. M. van Ruitenbeek, New J. Phys. 11, (2009) 073043.

[11] R. Suzuki, Y. Mukai, M. Tsutsui, S. Kurokawa, and A. Sakai, Jpn. J. Appl. Phys. 45, (2006) 7217.

[12] B. B. Lewis, K. G. Vandervoort, and R. D. Foster, Solid State Commun. 109, (1999) 525.

[13] A. Takahashi, S. Kurokawa, and A. Sakai, Phys. Status Solidi A 209, (2012) 2151.

[14] T. H. Courtney, Mechanical Behavior of Materials, Second Edition (McGraw-Hill, New York 2000).

[15] F. Cleri and V. Rosato, Phys. Rev. B 48, (1993) 22.

[16] P. García-Mochales, R. Paredes, S. Peláez, and P. A. Serena, Nanotechnology 19, (2008) 225704.

[17] S. Peláez, Guerrero C, R. Paredes, P. A. Serena, and P. García-Mochales, Electrodeposited Nanowires and Their Applications, Lupu N, ed. p.35 (INTECH, Croatia, 2010).

[18] G. M. Finbow, R. M. Lynden-Bell, and I. R. McDonald, Mol. Phys. 92, (1997) 705.

[19] A. Hasmy, E. Medina, and P. A. Serena, Phys. Rev. Lett. 86, (2001) 24.

[20] R. N. Barnett and U. Landman, Nature 387, (1997) 788.

[21] P. García-Mochales, R. Paredes, S. Peláez, and P. A. Serena, J. Nanomater. (2008) 1.

[22] P. García-Mochales, R. Paredes, S. Peláez, and P. A. Serena, Phys. Status Solidi (a) 205, (2008) 1317.

[23] S. Peláez, P. A. Serena, C. Guerrero, R. Paredes, and P. García-Mochales, Current Nanosicnece 7, (2011) 219.

[24] P. García-Mochales, S. Peláez, P. A. Serena, C. Guerrero, and R. Paredes, Modelling Simul. Mater. Sci. Eng. 21, (2013) 045002.

[25] V. K. Sutrakar and D. R. Mahapatra, J. Phys. Condens. Matter. 20, (2008) 335206.

[26] H. S. Park and J. A. Zimmerman, Scripta Mater. 54, (2006) 1127.

[27] H. Mehrez and S. Ciraci, Phys. Rev. B 56, (1997) 12632.

[28] O. Gülseren, F. Ercolessi, and E. Tosatti, Phys. Rev. Lett. 80, (1998) 3775.

[29] P. Sen, O. Gülseren, T. Yildirim, I. P. Batra, and S. Ciraci, Phys. Rev. B 65, (2002) 235433.

[30] J. H. Conway and N. J. A. Sloan, Sphere Packing, Lattices and Groups (A Series of Comprehensive Mathematical Studies vol 290) (Springer-Verlag, New York, 1999). 
[31] Y. Moriguchi, K. Yamauchi, S. Kurokawa, and A. Sakai, Surf. Sci. 606, (2012) 928.

[32] J. C. González, V. Rodrigues, J. Bettini, L. G.C. Rego, A. R. Rocha, P.Z. Coura, S. O. Dantas, F. Sato, D. S. Galvão, and D. Ugarte, Phys. Rev. Lett. 93, (2004) 3775. 